\title{
Trombiculidiasis en felino: reporte de un caso clínico
}

\author{
Meyer, S.N.; Benítez, J.S.; Maza, Y. \\ Cátedras de Semiología y Enfermedades Parasitarias, Facultad de Ciencias Veterinarias, Universidad \\ Nacional del Nordeste, Sargento Cabral 2139, Corrientes (3400), Argentina. \\ Tel. (0379) 425753. Int: 183. E-mail: semiol@vet.unne.edu.ar
}

\begin{abstract}
Resumen
Meyer, S.N.; Benítez, J.S.; Maza, Y.: Trombiculidiasis en felino: reporte de un caso clínico. Rev. vet. 25: 2, 158-160, 2014. La trombiculidiasis es una enfermedad parasitaria de perros, gatos, seres humanos y vertebrados en general, provocada por larvas de Trombicula autumnalis (Acarina: Trombiculidae), ácaro habitante del suelo y subsuelo, de aparición estacional. Enzimas proteolíticas de su saliva provocan una reacción de hipersensibilidad en la piel del huésped. El diagnóstico se confirma mediante raspado cutáneo superficial. El tratamiento debe orientarse a eliminar los parásitos del huésped y del ambiente. Se reporta un caso de trombiculidiasis en un felino que presentaba lesiones alopécicas, eritematosas y costrosas, así como su favorable respuesta al tratamiento instaurado. Se enfatiza la necesidad de combatir este parásito, habitualmente no tenido en cuenta, dado que es capaz de transmitir enfermedades infecciosas potencialmente mortales.
\end{abstract}

Palabras clave: gato, dermopatía, Trombicula autumnalis, tratamiento.

\begin{abstract}
.
Meyer, S.N; Benítez, J.S.; Maza, Y.: Trombiculidiasis in a feline: report of a case. Rev. vet. 25: 1, 158-160, 2014. Trombiculidiasis is a parasitic illness of dogs, cats, human beings and vertebrates, caused by larvae of Trombicula autumnalis, acarus of seasonal appearance that inhabits the soil and underground. Proteolytical enzymes of its saliva cause a hypersensitivity reaction in the guest's skin. Diagnosis is confirmed by means of a superficial skin scraping. Treatment should be guided to eliminate the parasites from the guests and the environment. A case of trombiculidiasis is reported in a feline that presented alopecic, erythematous and crusted lesions, as well as its favorable response to treatment. The necessity of combating the parasite, not usually considered as an etiology and being capable of transmitting potentially fatal infectious diseases is emphasized.
\end{abstract}

Key words: cat, dermopathy, Trombicula autumnalis, treatment.

\section{INTRODUCCIÓN}

La trombiculidiasis es una enfermedad parasitaria ocasional de perros, gatos, seres humanos o de cualquier otro animal vertebrado, provocada por larvas de diversas especies de Trombicula ${ }^{1,5,8}$. En Argentina, el agente causal es Trombicula autumnalis, también conocido como "bicho colorado" 8 .

Esta parasitosis es de aparición estacional, generalmente en verano y otoño. T. autumnalis es un ácaro, habitante del suelo y subsuelo, pudiendo alcanzar profundidades de hasta un metro cuando las condiciones climáticas son adversas, como puede ocurrir durante sequías, frío intenso o fuertes lluvias ${ }^{6,7}$. El ciclo biológico dura alrededor de 7 a 10 semanas y los ácaros pueden vivir más de un año ${ }^{4,8}$. En su mayor parte, el

Recibido: 4 septiembre 2014 / Aceptado: 3 octubre 2014 ciclo transcurre con estadios de vida libre. Los trombicúlidos adultos miden entre 1 y $2 \mathrm{~mm}$, tienen 8 patas, son de color rojo brillante y de apariencia aterciopelada. Se alimentan de otros ácaros, de insectos pequeños y de sus huevos, pudiendo devorar cantidades considerables de huevos de mosquitos Aedes ${ }^{4}$.

La hembra grávida coloca aproximadamente 40 huevos mensuales en el suelo, éstos se incuban por un período de 1 a 2 semanas, tras lo cual emerge la larva hexápoda, de 0,15 a $0,30 \mathrm{~mm}$ de largo, que es la forma parasitaria para el hombre y los animales ${ }^{9,12}$. Gracias a su fototropismo positivo, las larvas se concentran en la punta de las plantas y otros objetos, desde donde trepan hasta sus huéspedes, a los que detectan por el movimiento, dióxido de carbono, el olor y otros estímulos ${ }^{7}$. Las larvas pueden permanecer sobre el huésped varios días o hasta un mes ${ }^{4}$. Generalmente se desarrolla una sola generación de ácaros por año. 
Los lugares de preferencia sobre el animal son las patas, pabellón auricular, parte interna del conducto auditivo externo, zonas cercanas a los ojos, labios, dorso de la nariz, y además -en los gatos- la punta de la cola $^{8}$. Los animales pueden manifestar prurito intenso, aunque la larva ya no se encuentre; las lesiones más frecuentes son alopecia, descamación y erupción papular en patas y orejas. En gatos puede ocasionar una dermatitis miliar ${ }^{7,8}$.

Los quelíceros de las larvas rasguñan las capas superficiales de la piel y mediante su saliva cargada con enzimas proteolíticas, desintegran las células de los estratos espinoso y germinativo y las ingieren por succión; no son hematófagas, a pesar de lo que pueda creerse por su tonalidad rojiza. Esta acción provoca sobre el huésped una reacción de hipersensibilidad ${ }^{4}$. La anamnesis reviste fundamental importancia durante la exploración clínica, ya que la sintomatología es muy variable y el conocimiento del hábitat y hábitos del animal, orientan hacia el diagnóstico de esta enfermedad. El diagnóstico definitivo se logra mediante la observación de la larva del ácaro en un raspado superficial de piel ${ }^{8}$.

El tratamiento inicial de la afección está orientado a eliminar el parásito, aunque su débil fijación lo hace susceptible de desprendimiento por el rascado. Existe escaso material referido al tratamiento farmacológico para la eliminación de las larvas, pero en términos generales se recomiendan productos que contengan piretroides, fosforados o fipronil. Algunos autores destacan también la eficacia de la ivermectina subcutánea ${ }^{2,10} \mathrm{y}$ otros pediculicidas en perros ${ }^{11}$. Una vez que el parásito se ha desprendido, la terapia se orienta a la eliminación del prurito, siendo recomendable la utilización de glucocorticoides ${ }^{1,12}$.

La prevención radica en evitar las áreas infestadas. La eliminación de los ácaros en su hábitat es dificultosa, debido a su distribución en parches ${ }^{9}$. De ser posible la identificación de los parches de vegetación, que usualmente albergan gran número de larvas, puede ser ventajosa su eliminación mediante el desmalezado, limpieza y fumigación del espacio verde, con productos insecticidas.

El objetivo del presente trabajo fue reportar un caso clínico de esta enfermedad parasitaria que habitualmente no es tenida en cuenta, pero que reviste importancia sanitaria, no solo para los animales domésticos, sino también para el hombre.

\section{MATERIAL Y MÉTODOS}

Se trabajó con un paciente felino, de raza indefinida, hembra, castrada, de 3 años de edad, proveniente de la zona de Puerto Tirol, Provincia del Chaco. La paciente ingresó a la consulta en el mes de octubre, con prurito intenso en las regiones de la cabeza y cuello, donde -a través de la inspección- se apreciaron lesiones alopécicas, eritematosas, costrosas y escoriaciones difusas. Alrededor de las lesiones, adherida a los pelos, presentó una leve descamación furfurácea (Figura 1).
La sintomatología se había iniciado entre los meses de marzo y abril. Por anamnesis se pudo conocer que la paciente convivía con otros felinos y caninos, que no se encontraban afectados, así como tampoco los propietarios. Todos los animales de la casa compartían el mismo ambiente, pero la paciente era la única que tenía el hábito de acostarse sobre el césped, muy próxima a plantas ornamentales de salvia, aloe vera, helechos y un árbol de fresno.

Efectuadas la inspección y la palpación, como método complementario de diagnóstico se optó por el raspado de piel, utilizando una hoja de bisturí, depositando el material sobre un portaobjeto con unas gotas de vaselina liquida, utilizando guantes descartables. En primera instancia, la muestra se obtuvo de una lesión eritematosa, de apariencia incipiente, sin costra; el resultado de la misma fue negativo. Las muestras subsiguientes fueron tomadas de lesiones costrosas, en las que se pudieron visualizar las larvas del parásito por microscopía óptica (Figura 2).

Una vez identificado el agente, se procedió a rasurar al animal con una máquina peladora, situación que evidenció una mayor extensión y profundidad de las lesiones; las mismas se extendían desde la cara hasta la región de la cruz, sin afectar a los miembros ni el resto del cuerpo (Figura 3). Con los datos obtenidos se arribó al diagnóstico de trombiculidiasis y se instauró el tratamiento parasitológico (sobre el ambiente y sobre el animal) y de las heridas de la piel.

El tratamiento consistió en fumigaciones con un producto a base de cipermetrina y butóxido de piperonilo, una vez cada quince días, para combatir los esta-

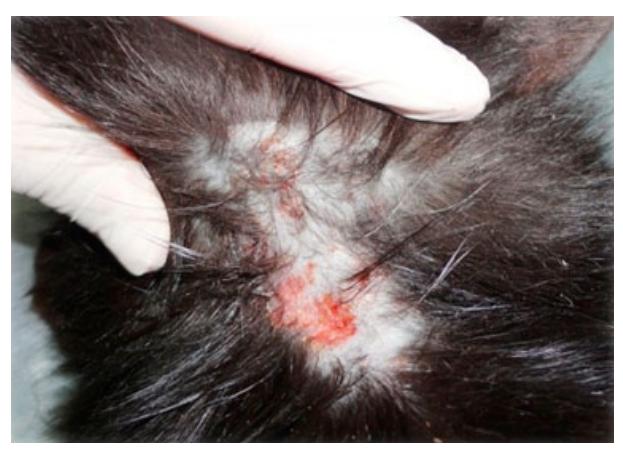

Figura 1. Lesiones de piel al momento de la consulta.

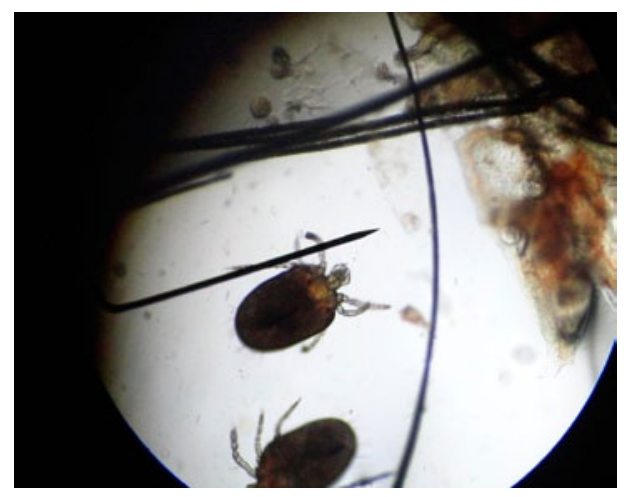

Figura 2. Larva hexápoda de T. autumnalis. 


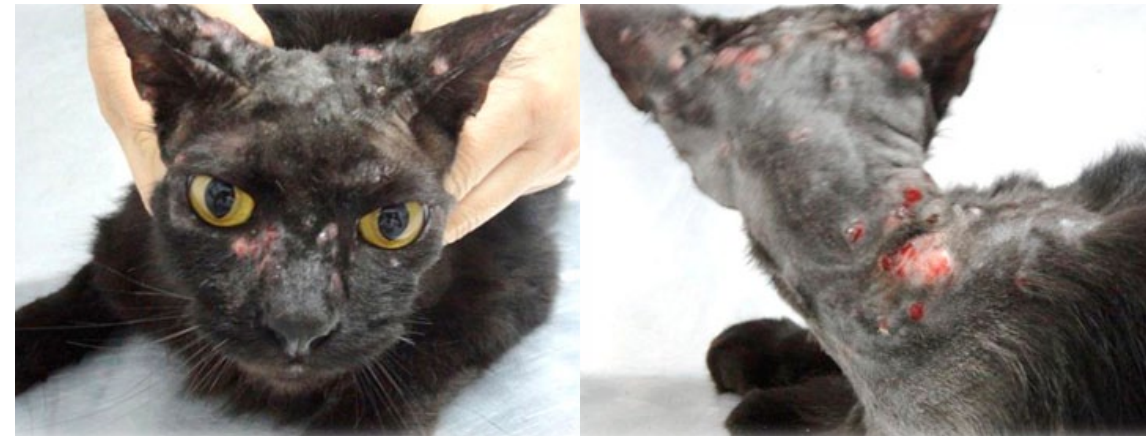

Figura 3. Extensión de las lesiones cefálicas evidenciadas por tricotomía.

dios adultos, mientras que para la paciente se indicaron cuatro aplicaciones de pipetas con fipronil, a intervalos de quince días cada una, a fin de combatir las formas larvarias, además de una única dosis de ivermectina por vía oral, al momento del diagnóstico. Las lesiones de piel fueron tratadas con antibióticos, antiinflamatorios y pomadas cicatrizantes. Se colocó collar isabelino para evitar la progresión de las lesiones por el rascado.

\section{RESULTADOS Y DISCUSIÓN}

Si bien el prurito disminuyó, el alivio total del mismo se logró recién al momento en que la propietaria pudo comenzar con el tratamiento ambiental, lo que no fue coincidente con el inicio del tratamiento antiparasitario sobre el animal.

Después de iniciado el tratamiento la paciente se recuperó favorablemente. Sin embargo, la propietaria informó sobre la aparición esporádica de nuevas lesiones y episodios de prurito alrededor de los pabellones auriculares, situación que ella controló con la utilización del producto mencionado, pulverizando el ambiente y topicando con un algodón sobre el animal. Esto último no fue recomendado por el profesional actuante.

La trombiculidiasis es una enfermedad de escasa aparición en los registros de lesiones dermatológicas en pequeños animales. Las lesiones que provoca y la intensidad del prurito hacen necesaria su consideración como parte del diagnóstico diferencial con otras enfermedades parasitarias pruriginosas, tales como la escabiosis $^{3}$.

Las lesiones encontradas, coinciden con las descriptas anteriormente para esta parasitosis ${ }^{8}$, excepto en lo referente a su ubicación a nivel de las patas, punta de la cola y el patrón de dermatitis miliar. En este caso se hallaron a nivel de la línea dorsal, desde la nariz hasta la cruz y predominantemente en forma de placas, lo que podría indicar la reacción de hipersensibilidad que provoca sobre el animal, acorde a lo descrito por otros autores ${ }^{4}$.

Respecto al sitio de elección para el raspado, llamó la atención la obtención de muestras positivas solamente en las lesiones costrosas, contrariamente a lo que habitualmente se recomienda al momento de obtener muestras por raspado de piel, a partir de la periferia de las lesiones recientes.
La bibliografía aporta pocos datos respecto al tratamiento de esta enfermedad, quizás por su escasa frecuencia de aparición en nuestra zona o por no ser tenida en cuenta al momento de diagnosticar enfermedades parasitarias de la piel. Es importante tratar al ambiente $\mathrm{y}$ al paciente, por las características que presenta el ciclo de vida del parásito $\mathrm{y}$, acorde con algunos especialistas ${ }^{7}$, constituye la única manera de lograr el éxito terapéutico.

A pesar de que en Argentina no se encuentran datos, en otros continentes existen especies de trombicúlidos responsables de la transmisión de enfermedades infecciosas potencialmente mortales como rickettsiosis, borreliosis y ehrlichiosis ${ }^{2}$, lo que demuestra la importancia de considerar su estudio a futuro.

\section{REFERENCIAS}

1. Angel TA, Nigro J, Levy ML. 2000. Infestations in the pediatric patient. Pediatr Clin North Am 47: 921-935.

2. Beltrán M, Valdivia C, Ponce R, Chambergo M. 2009. Trombicula autumnalis in kindergarten children from Peruvian jungle. Rev Per Med Exp 26: 58-60.

3. Chaccour CJ. 2005. Trombiculiasis: reporte de dos casos y revisión de la literatura. Dermatol Venez 43: 18-21.

4. Chandler AC, Read CP. 1965. Introducción a la Parasitología, Ed. Omega, Barcelona, p. 569-574.

5. Durden LA, Ellis BA, Banks CW, Crowe JD, Oliver JH. 2004. Ectoparasites of gray squirrels in two different habitats and screening of selected ectoparasites for bartonellae. J Parasitol 90: 485-489.

6. Gentry JW, Phang OW, Manikumaran C. 1977. The effects of rainfall on trombiculid (Acarina: trombiculidae) larval populations in peninsular Malaysia. Southeast Asian J Trop Med Public Health 8: 217-220.

7. Georgi JR, Georgi ME. 1994. Parasitología en Clínica Canina, Ed. Interamericana, México, p. 55-58.

8. Pérez Tort G, Welch EL. 1998. Enfoque clínico de las enfermedades parasitarias de los perros y gatos, Ed. Agrovet, Buenos Aires, p. 249-250.

9. Rozendal J. 1997. Vector control: methods for use by individuals and communities. WHO. http://www.who.int/ water_sanitation_health/resources/vectorcontrol/en/

10. Sladky KK, Norton TM, Loomis MR. 2000. Trombiculid mites (Hannemania sp.) in canyon tree frogs (Hyla arenicolor). J Zoo Wildl Med 31: 570-575.

11. Smal D, Jasmin P, Mercier P. 2004. Treatment of Neotrombicula autumnalis dermatitis in dogs using two topical permethrin-pyriproxyfen combinations. J Small Anim Pract 45: 98-103.

12. Steen CJ, Carbonaro PA, Schwartz RA. 2004. Arthropods in dermatology. J Am Acad Dermatol 50: 819-842. 